\title{
Gd(III) Chelates as NMR Probes of Protein-Protein Interactions. Case Study: Rubredoxin and Cytochrome $c_{3}$
}

\author{
Rui M. Almeida, ${ }^{\dagger}$ Carlos F. G. C. Geraldes, ${ }^{\ddagger}$ Sofia R. Pauleta, ${ }^{*,+}$ and José J. G. Moura ${ }^{*,+}$ \\ ${ }^{\dagger}$ REQUIMTE/CQFB, Departamento de Química, Faculdade de Ciências e Tecnologia, Universidade Nova de Lisboa, \\ 2829-516 Caparica, Portugal \\ ${ }^{\ddagger}$ Department of Life Sciences, Faculty of Science and Technology and Centre of Neurosciences and Cell Biology, University of Coimbra, \\ 3001-401 Coimbra, Portugal
}

Supporting Information

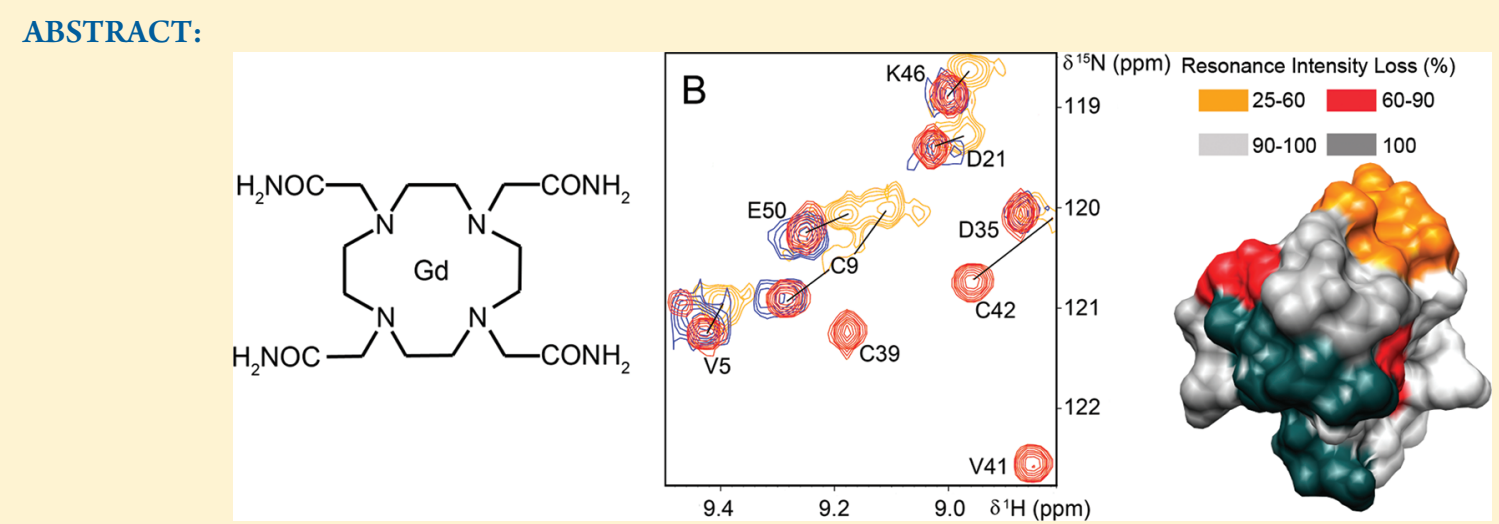

Two cyclen-derived Gd probes, $[\mathrm{Gd}-\mathrm{DOTAM}]^{3+}$ and $[\mathrm{Gd}-\mathrm{DOTP}]^{5-}(\mathrm{DOTAM}=1,4,7,10$-tetraazacyclododecane-1,4,7,10tetraacetamide; DOTP = 1,4,7,10-tetraazacyclododecane-1,4,7,10-tetrakis(methylenephosphonate)), were assessed as paramagnetic relaxation enhancement (PRE)-inducing probes for characterization of protein-protein interactions. Two proteins, Desulfovibrio gigas rubredoxin and Desulfovibrio gigas cytochrome $c_{3}$, were used as model partners. In a ${ }^{1} \mathrm{H}$ NMR titration it was shown that $[\mathrm{Gd}-\mathrm{DOTP}]^{5-}$ binds to cytochrome $c_{3}$ near heme IV, causing pronounced PREs, characterized by line width broadenings of the heme methyl resonances at ratios as low as 0.08 . A $K_{\mathrm{d}}$ of $23 \pm 1 \mu \mathrm{M}$ was calculated based on chemical shift perturbation of selected heme methyl resonances belonging to three different heme groups, caused by allosteric effects upon $[\mathrm{Gd}-\mathrm{DOTP}]^{5-}$ binding to cytochrome $c_{3}$ at a molar ratio of 2 . The other probe, $[\mathrm{Gd}-\mathrm{DOTAM}]^{3+}$, caused PREs on a well-defined patch near the metal center of rubredoxin (especially the patch constituted by residues D19-G23 and W37-S45, which broaden beyond detection). This effect was partially reversed for some resonances (C6-Y11, in particular) when cytochrome $c_{3}$ was added to this system. Both probes were successful in causing reversible PREs at the partner binding site, thus showing to be good probes to identify partners' binding sites and since the interaction is reversible to structurally characterize protein complexes by better defining the complex interface.

\section{INTRODUCTION}

The paramagnetic trivalent lanthanide ions constitute a series with similar chemical behavior but very different physical properties as a result of their partially filled $4 \mathrm{f}$ electron shell. Therefore, each trivalent lanthanide ion exhibits different characteristic effects on the NMR parameters of a nucleus in their proximity. Such perturbations, including lanthanide-induced shifts (LIS) and paramagnetic relaxation enhancements (PRE), have been extensively exploited for elucidation of molecular structures, including those of proteins and nucleic acids in solution. ${ }^{1-3}$

When a Lewis base interacts with a $\mathrm{Ln}^{3+}$ ion, its NMR-active nuclei are influenced by the presence of the unpaired $\mathrm{f}$ electrons, leading in some cases to a shift to a different NMR frequency but always to a paramagnetic relaxation enhancement and broadening of its resonance. The paramagnetic $\mathrm{Ln}^{3+}$ ions (except $\mathrm{Gd}^{3+}$ ), due to the anisotropic spatial distribution of unpaired $\mathrm{f}$ electrons, originate a dipolar LIS in solution, called pseudocontact shift (PCS), which is useful to obtain structural information. If, however, the Lewis acid-base interaction is partially covalent, a small amount of the unpaired electron spin density can reach the molecular framework of the base and originate a second type of LIS, the contact (or scalar) shift.

Received: April 24, 2011

Published: September 29, 2011 
The pseudocontact or dipolar shift (PCS) is due to the local magnetic field induced in the observed nucleus by the anisotropy of the magnetic susceptibility tensor $\chi$ of the lanthanide ion. Since the PCS depends on $1 / r^{3}$, it is observable for nuclei at relatively long distances; for certain lanthanides with larger magnetic moments, such as $\mathrm{Dy}^{3+}$, and depending on the relative orientation of the lanthanide ion magnetic susceptibility tensor to the nucleus under observation, PCS effects can be observed at distances of up to $40 \AA$ from the paramagnetic center.,

The lanthanide ion $\mathrm{Gd}^{3+}$ has 7 unpaired electrons distributed isotropically throughout its $4 \mathrm{f}$ shell and therefore cannot produce a NMR dipolar shift in solution. Nevertheless, it is highly efficient in causing nuclear relaxation, which led to the successful use of $\mathrm{Gd}^{3+}$. based contrast agents in MRI (magnetic resonance imaging). ${ }^{3}$

Paramagnetic relaxation enhancements by $\mathrm{Gd}^{3+}$ are dominated by the dipolar contribution for all nuclei except those directly bound and can be described by the Solomon-Bloembergen equations (eqs 1 and 2), assuming that the complexes undergo isotropic reorientation

$$
\frac{1}{T_{1, \mathrm{dip}}}=\frac{2}{15}\left(\frac{\mu_{0}}{4 \pi}\right)^{2} \frac{\gamma_{\mathrm{I}}^{2} \mu_{\mathrm{eff}}^{2}}{r^{6}}\left(\frac{3 \tau_{c 1}}{1+\omega_{\mathrm{I}}^{2} \tau_{\mathrm{c} 1}^{2}}+\frac{7 \tau_{\mathrm{c} 2}}{1+\omega_{\mathrm{S}}^{2} \tau_{\mathrm{c} 2}^{2}}\right)
$$

$$
\frac{1}{T_{2, \text { dip }}}=\frac{1}{15}\left(\frac{\mu_{0}}{4 \pi}\right)^{2} \frac{\gamma_{\mathrm{I}}^{2} \mu_{\mathrm{eff}}^{2}}{r^{6}}\left(4 \tau_{\mathrm{c} 1}+\frac{3 \tau_{\mathrm{c} 1}}{1+\omega_{\mathrm{I}}^{2} \tau_{\mathrm{c} 1}^{2}}+\frac{13 \tau_{\mathrm{c} 2}}{1+\omega_{\mathrm{S}}^{2} \tau_{\mathrm{c} 2}^{2}}\right)
$$

where $\mu_{0} / 4 \pi$ is the magnetic permeability of vacuum, $\mu_{\mathrm{eff}}$ is the effective magnetic moment of $\mathrm{Gd}^{3+}, \omega_{\mathrm{I}}$ and $\omega_{\mathrm{S}}$ are the Larmor frequency of the nucleus under study and of the electron spin, respectively, and $\tau_{\mathrm{c} i}(i=1,2)$ is given by

$$
\frac{1}{\tau_{\mathrm{ci}}}=\frac{1}{T_{i \mathrm{e}}}+\frac{1}{\tau_{\mathrm{M}}}+\frac{1}{\tau_{\mathrm{R}}}
$$

where $\tau_{\mathrm{R}}$ is the rotational tumbling time of the complex, $\tau_{\mathrm{M}}$ is the chemical exchange time, and $T_{i \mathrm{e}}$ are the longitudinal and transverse electronic relaxation times. The relaxationenhancing effect diminishes steeply upon increase of the distance from the lanthanide ion due to its $1 / r^{6}$ dependence. However, at short distances it leads to dramatic line width broadening (up to $0.040 \%$ of the Larmor frequency for a proton at $5 \AA$ from the paramagnetic source ${ }^{1}$ ), but unlike other lanthanides, it does not originate any significant PCS, residual dipolar couplings (RDC), or cross-correlation rate (CCR) effects. This characteristic is fundamental for accurate distance-dependent measurements. 5

Furthermore, $\mathrm{Gd}^{3+}$ compounds can also be used as primers for paramagnetism-based NMR structure calculation experiments by facilitating determination of the metal position relative to the macromolecule under study, which will assist in analysis of the spectral aspects induced by the magnetic susceptibility anisotropy of other lanthanides. 6,7 Consequently, relaxation data are particularly powerful in obtaining the geometrical parameters of a nucleus in the proximity of the $\mathrm{Gd}^{3+}$ ion.

Early use of $\mathrm{Ln}^{3+}$ ions as NMR structural probes of proteins in aqueous solution using PCS and PRE effects has been reviewed. ${ }^{8}$ More recently, these paramagnetic structural constraints were used together with RDCs and CCRs induced by $\mathrm{Ln}^{3+}$ different from $\mathrm{Gd}^{3+}$ to better define the structure of

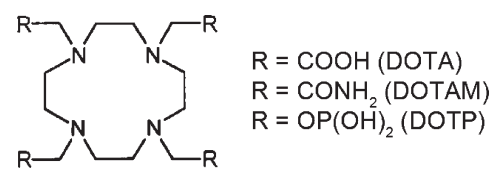

Figure 1. General structure of the DOTA-like (tetrakis)-N-substituted cyclen ligands with common substituents and acronyms listed.

lanthanide-substituted proteins, such as the $\mathrm{Ca}^{2+}$-binding proteins calbindin $\mathrm{D}_{9 \mathrm{k}}$ and calmodulins. ${ }^{4,9-18}$ When an intrinsic diamagnetic metal substitution is not possible or cannot be engineered inside the core of a given protein, paramagnetic probes or tags can be constructed for covalent attachment to the protein in order to weakly orient it in a magnetic field, yielding PCS and RDCs. ${ }^{19-27}$

An approach to study protein-protein interactions, which does not need generation of protein mutants to covalently bind paramagnetic tags, is the use of cosolute paramagnetic complexes. ${ }^{28}$ Recognition of a protein surface by another surface is a molecular process largely dependent on long-range electrostatic forces that make the surfaces approach each other, but its selectivity is mainly driven by very specific short-range hydrophobic effects. The potential energy surfaces of the interacting proteins depend on the polarity, size, shape, and flexibility of their surfaces, which are determined by the geometric distribution of cationic and anionic surface residues, which are concentrated in charged patches, ${ }^{29}$ in many cases surrounding hydrophobic patches.

Studies of the noncovalent binding of small charged cationic and anionic paramagnetic chelates to protein surfaces can be used as a tool to study protein surface recognition by NMR. One of those case studies involved cytochrome $c$ and use of small complexes of different sizes, shapes, and charges as paramagnetic NMR shift and/or relaxation agents. Analysis of the localization of their binding sites was possible through observation of the NMR effects on specific resonances, which functioned as reporter groups. ${ }^{30,31}$

The specificity of most of the neutral or low charged $\mathrm{Ln}^{3+}$ complexes used as NMR probes (such as [Gd(DTPA-BMA)$\left.\left(\mathrm{H}_{2} \mathrm{O}\right)\right],\left[\operatorname{Ln}(\mathrm{DOTA})\left(\mathrm{H}_{2} \mathrm{O}\right)\right]^{-}$, and $\left.\left[\operatorname{Ln}(\mathrm{DTPA})\left(\mathrm{H}_{2} \mathrm{O}\right)\right]^{-}\right)$is quite limited, ${ }^{13,32-35}$ and the structural information obtained is limited due to the small PCS values that result from averaging effects. However, the probe specificity can be increased by increasing the number of inner-sphere free sites to two for direct binding to surface side-chain residues, like in $[\mathrm{Gd}(\mathrm{DO} 3 \mathrm{~A})$ $\left.\left(\mathrm{H}_{2} \mathrm{O}\right)_{2}\right]$, which was found to bind calbindin $\mathrm{D}_{9 \mathrm{k}}$ at four preferential carboxylate-rich surface sites. ${ }^{36}$ On the other hand, Otting and co-workers recently characterized a dipicolinic acidderived lanthanide chelator $\left(\left[\operatorname{Ln}(\mathrm{DPA})_{3}\right]^{3-}\right)$ that can bind specifically to protein sites where an arginine residue is present. ${ }^{15}$

In this work, we tested the possibility of increasing the surface probe specificity by increasing its negative or positive charge in order to act as a strong outer-sphere chelator $\left([\operatorname{Ln}(\mathrm{DOTP})]^{5-}\right.$, $\mathrm{H}_{8} \mathrm{DOTP}=1,4,7,10$-tetraazacyclododecane-1,4,7,10-tetrakis(methylenephosphonic acid), and $\left[\mathrm{Ln}(\right.$ DOTAM $\left.)\left(\mathrm{H}_{2} \mathrm{O}\right)^{3+}\right]$, DOTAM = 1,4,7,10-tetraazacyclododecane-1,4,7,10-tetraacetamide) and using $\mathrm{Gd}^{3+}$ as the chelated lanthanide ion, as PRE has a shorter range than PCS. The $[\operatorname{Ln}(\mathrm{DOTP})]^{5-}$ probes were found to preferentially bind to lysyl residues. ${ }^{37,38}$ Thus, two DOTA-like $\mathrm{Gd}^{3+}$ chelates with opposite charges, $[\mathrm{Gd}(\mathrm{DOTP})]^{5-}$ and $\left[\mathrm{Gd}(\mathrm{DOTAM})\left(\mathrm{H}_{2} \mathrm{O}\right)^{3+}\right]$ (Figure 1), were used as NMR paramagnetic relaxation probes to 

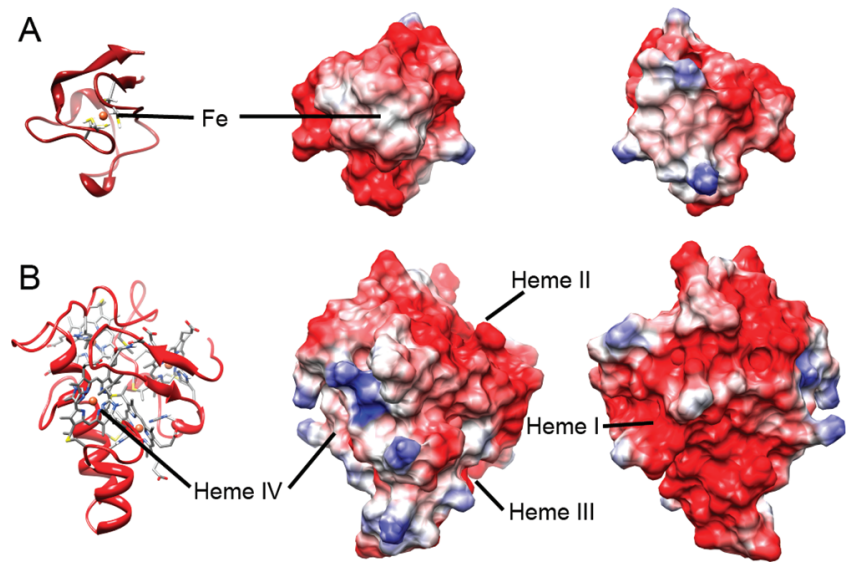

Figure 2. (A) (Left) Ribbon representation of D. gigas rubredoxin (PDB code 1RDG). (Middle) Coulombic surface coloring of rubredoxin in the same orientation as in the left-hand-side panel. (Right) $180^{\circ}$ rotation on the vertical axis of the middle panel. (B) (Left) Ribbon representation of $D$. gigas cytochrome $c_{3}$. (Middle) Coulombic surface coloring of cytochrome $c_{3}$ in the same orientation as in the left-hand-side panel. (Right) $180^{\circ}$ rotation on the vertical axis of the middle panel. (Blue) Positively charged residues (most intense $=+10 \mathrm{kcal} \mathrm{mol}^{-1} \cdot \mathrm{e}$ ). (Red) Negatively charged residues (most intense $=-10 \mathrm{kcal} \mathrm{mol}^{-1} \cdot \mathrm{e}$ ). (White) Neutral residues. Images created with UCSF Chimera. ${ }^{55}$ Coulombic charges calculated using the Amber force field ff99SB package for the aminoacid residues ${ }^{56}$ and ANTECHAMBER for all other charges. ${ }^{57}$

characterize the interaction with two well-studied proteins: Desulfovibrio (D.) gigas rubredoxin and D. gigas cytochrome $c_{3}{ }^{28}$

These proteins can be considered as models for other FeS centers or heme proteins, respectively, and have characteristics complementary to one another. While rubredoxin is essentially acidic at physiological $\mathrm{pH}(\mathrm{pI} \approx 4)$ with a hydrophobic patch in the vicinity of the metal center, the environment around the four heme groups of cytochrome $c_{3}$ can vary between negatively charged and positively charged, though this protein is also acidic $(\mathrm{pI} \approx 5)$. These different charged patches create a range of different conditions that can model the binding of each Gdchelate probe (Figure 2). In addition, the paramagnetic relaxation probes were used to obtain information on the interface of the well-characterized complex between rubredoxin and cytochrome $c_{3}{ }^{28}$ by comparing the PREs obtained by the probe on the protein alone with those obtained on the complex formed with its partner.

\section{EXPERIMENTAL SECTION}

Protein Purification. D. gigas $\mathrm{Zn}$-substituted ${ }^{15} \mathrm{~N}$-rubredoxin and D. gigas cytochrome $c_{3}$ were isolated as previously described in ref 28 .

Gd-Probe Preparation. The macrocyclic ligands DOTAM and $\mathrm{H}_{8}$ DOTP and their $\mathrm{Gd}^{3+}$ complexes were synthesized according to published procedures. ${ }^{39-42}$ The amount of free $\mathrm{Gd}^{3+}$ in the final solutions was estimated to be less than $4 \%$ of the total amount. ${ }^{43}$

NMR Spectroscopy. (i). Sample Preparation. In the ${ }^{1} \mathrm{H}$ NMR experiments, aliquots of either $[\mathrm{Gd}-\mathrm{DOTAM}]^{3+}$ or $[\mathrm{Gd}-\mathrm{DOTP}]^{5-}$ (at $2.4 \mathrm{mM}$ concentration in water, with $\mathrm{pH}$ adjusted to 7.0 ) were added to a $0.1 \mathrm{mM}$ solution of cytochrome $c_{3}$ in $10 \mathrm{mM}$ Tris- $\mathrm{HCl} \mathrm{pH} 7.6$ and $10 \% \mathrm{D}_{2} \mathrm{O}$. In the 2D NMR experiments, aliquots of the same solution of either $[\mathrm{Gd}-\mathrm{DOTAM}]^{3+}$ or $[\mathrm{Gd}-\mathrm{DOTP}]^{5-}$ at $2.4 \mathrm{mM}$ concentration were added to a $0.1 \mathrm{mM}$ solution of ${ }^{15} \mathrm{~N}-\mathrm{Zn}$-rubredoxin in $10 \mathrm{mM}$ Tris- $\mathrm{HCl} \mathrm{pH} 7.6$ and $10 \% \mathrm{D}_{2} \mathrm{O}$ until a ratio of [Gd probe]/[rubredoxin] of 0.5 for $[\mathrm{Gd}-\mathrm{DOTP}]^{5-}$ or 2.0 in the case of $[\mathrm{Gd}-\mathrm{DOTAM}]^{3+}$.
After the $[\mathrm{Gd}-\mathrm{DOTAM}]^{3+}$-rubredoxin titration reached a $1: 2$ molar ratio, aliquots of cytochrome $c_{3}$ were added until a final molar ratio of $1: 2: 2$.

(ii). Data Acquisition. 1D NMR spectra were recorded using presaturation of water with a spectral width of $60 \mathrm{ppm}$ for $32 \mathrm{k}$ data points and 1024 scans accumulated. To improve the signal-to-noise ratio, an exponential multiplication by $10 \mathrm{~Hz}$ line broadening of the free induction decay was applied prior to Fourier transformation. ${ }^{1} \mathrm{H}-{ }^{15} \mathrm{~N}$ HSQC spectra were obtained using a phase-sensitive and water flip-back pulse sequence. The spectral widths are $7200 \mathrm{~Hz}$ for ${ }^{1} \mathrm{H}$ and $2430 \mathrm{~Hz}$ for ${ }^{15} \mathrm{~N}$. A total of 2048 data points in $t_{2}$ and 4 transients for each of the $256 t_{1}$ were used. NMR spectra were processed with TOPSPIN 2.1 provided by Bruker. ${ }^{15} \mathrm{~N}$ relaxation measurements $\left(\mathrm{R}_{1}, \mathrm{R}_{2}\right.$, and steady-state ${ }^{1} \mathrm{H}-{ }^{15} \mathrm{~N}$ NOE) were acquired using two-dimensional heteronuclear NMR experiments using standard pulse sequences. These experiments were performed for the following mixtures: $[\mathrm{Gd}-\mathrm{DOTAM}]^{3+}$-rubredoxin at 2:1, cytochrome $c_{3}$-rubredoxin at $2: 1$, and [Gd-DOTAM $]^{3+}-$ cytochrome $c_{3}$-rubredoxin at 2:2:1. Relevant spectra were acquired using 13 different delays ranging from 5 to $1000 \mathrm{~ms}$ (inversion recovery, for $\mathrm{R}_{1}$ ) or 14 different time delays ranging from 16 to $256 \mathrm{~ms}$ (CPMG (Carr-Purcell-Meiboom-Gill) pulses, for $\mathrm{R}_{2}$ ). All spectra were recorded at $298 \mathrm{~K}$ on a Bruker Avance III $600 \mathrm{MHz}$ spectrometer equipped with a TCI cryoprobe and a variable-temperature control unit and processed using TOPSPIN 2.1 by Bruker. ${ }^{1} \mathrm{H}$ chemical shifts were referenced to the $\mathrm{H}_{2} \mathrm{O}$ resonance ( $4.76 \mathrm{ppm}$ at $298 \mathrm{~K}$ ), and ${ }^{15} \mathrm{~N}$ chemical shifts were referenced indirectly using the above ${ }^{1} \mathrm{H}$ reference and gyromagnetic ratios (0.101329118).

(iii). Data Analysis. The chemical shift difference of the cytochrome $c_{3}$ heme methyls upon binding of $\mathrm{Gd}$ probe was analyzed considering a model for two identical binding sites. The chemical shift difference was nonlinear least-squares fitted using eq 4 , considering a fast exchange equilibrium

$$
\Delta \delta_{\mathrm{obs}}=\Delta \delta_{\max } x_{\mathrm{AB}}
$$

in which $x_{\mathrm{AB}}=[\mathrm{AB}] /[\mathrm{A}]_{T}$ and $[\mathrm{AB}]$ is given by $[\mathrm{AB}]=\left(K_{\mathrm{d}}+[\mathrm{A}]_{T}+\right.$ $\left.[\mathrm{B}]_{T}\right)-\left(\left(K_{\mathrm{d}}+[\mathrm{A}]_{T}+[\mathrm{B}]_{T}\right)^{2}-4 \cdot[\mathrm{A}]_{T}[\mathrm{~B}]_{T}\right)^{1 / 2} / 2 . K_{\mathrm{d}}$ and $\Delta \delta_{\max }$ were obtained simultaneously by nonlinear least-squares fitting the chemical shift difference using eq 4.

The $D$. gigas cytochrome $c_{3}$-induced changes in amide peak position of $\mathrm{Zn}$-rubredoxin, $\Delta \delta_{\mathrm{HN}}$ (in ppm), were reported as a combination of the changes in the proton $(\Delta \delta \mathrm{H})$ and nitrogen $(\Delta \delta \mathrm{N})$ dimensions according to the following equation $\Delta \delta \mathrm{HN}=\left(\left[(\Delta \delta \mathrm{H})^{2}+(\Delta \delta \mathrm{N} / 5)^{2}\right] / 2\right)^{1 / 2} \cdot{ }^{44}$

In the case of ${ }^{15} \mathrm{~N}$ relaxation experiments, peak integrals were calculated using Topspin and the time constant of their intensity decay was calculated using nonlinear least-squares fitting following equations of the type $I=I_{0} \cdot \mathrm{e}^{-t \cdot \mathrm{R} 1}+A$ or $I_{0} \cdot \mathrm{e}^{-t \cdot \mathrm{R} 2}$ for $\mathrm{R}_{1}$ and $\mathrm{R}_{2}$, respectively, where $I$ represents the peak integral, $I_{0}$ the peak integral at $t=0$, and $t$ the incremental time delay. ${ }^{45}$ The paramagnetic relaxation enhancement rate was measured as the difference in transverse relaxation rates between the paramagnetic, in the presence of Gd probe $\left(R_{2, \text { para }}\right)$, and diamagnetic $\left(R_{2, \text { dia }}\right)$, in the absence of $\mathrm{Gd}$ probe, states of $\mathrm{Zn}$-substituted rubredoxin (eq 5 )

$$
\Gamma_{2}=R_{2, \text { para }}-R_{2, \text { dia }}
$$

\section{RESULTS AND DISCUSSION}

Cytochrome $c_{3}$ : A Negatively Charged Protein with 4 c-Type Heme Groups. D. gigas cytochrome $c_{3}$, which at the working $\mathrm{pH}$ exhibits surface patches charged either positively (mostly around heme IV but also near heme III) or negatively (around hemes I and II, Figure 2), can be used as a suitable model protein in order to assess the feasibility of using [GdDOTAM $]^{3+}$ or $[\mathrm{Gd}-\mathrm{DOTP}]^{5-}$ as protein interaction probes. 


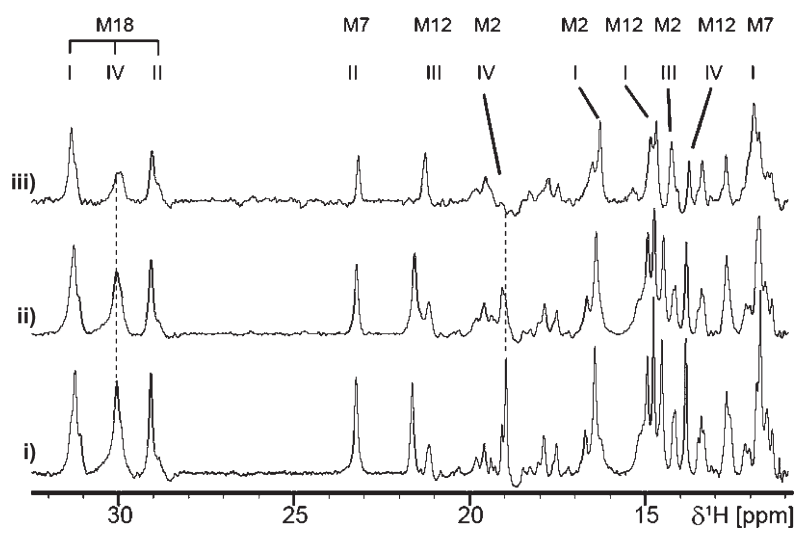

Figure 3. ${ }^{1} \mathrm{H}$ NMR spectra of the titration of $0.1 \mathrm{mM} \mathrm{D}$. gigas cytochrome $c_{3}$ with $[\mathrm{Gd}-\mathrm{DOTP}]^{5-}$ at different ratios. Heme methyl resonances were assigned as in ref 28: (i) $[\mathrm{Gd}-\mathrm{DOTP}]^{5-} /[$ cytochrome $\left.c_{3}\right]=0$, (ii) $[\mathrm{Gd}-\mathrm{DOTP}]^{5-} /\left[\right.$ cytochrome $\left.c_{3}\right]=0.08$, and (iii) $[\mathrm{Gd}-\mathrm{DOTP}]^{5-} /\left[\right.$ cytochrome $\left.c_{3}\right]=2$.

Titration of cytochrome $c_{3}$ with the positively charged probe did not yield significant effects on cytochrome $c_{3}$ heme methyl resonances: neither line width broadenings nor chemical shifts that could arise from conformational changes (see below; data not shown). This result indicates that there is no interaction between $[\mathrm{Gd}-\mathrm{DOTAM}]^{3+}$ and the heme groups of cytochrome $c_{3}$. This can be explained considering that the expected electrostatic attractions exerted by the negatively charged exposed propionate side chains of the heme groups, other than heme IV, are being attenuated by unfavorable interactions with other positively charged patches in their vicinity or steric hindrance is being imposed on the somewhat bulky chelate.

However, binding of $[\mathrm{Gd}-\mathrm{DOTAM}]^{3+}$ to the cytochrome $c_{3}$ surface away from the hemes cannot be ruled out, which would prevent observation of the expected paramagnetic effects on the heme methyl resonances. In fact, a docking study performed with Autodock Vina ${ }^{46}$ using the crystallographic structures of Gd-DOTAM and cytochrome $c_{3}$ as inputs does not succeed in placing the $\mathrm{Gd}^{3+}$ ion closer than $7 \AA$ to any methyl group from any of the 4 hemes. In fact, the minimum distance for most of the solutions obtained was between 8 and $9 \AA$ (data not shown), a distance too large for PREs to be detected in the heme methyl resonances.

Therefore, since cytochrome $c_{3}$ was chosen as a probe for studying the feasibility of $\mathrm{Gd}$ chelates as paramagnetic relaxation enhancers for (multi)heme proteins and specifically for the model complex with rubredoxin, no subsequent studies were performed on the cytochrome $c_{3}-[\mathrm{Gd}-\mathrm{DOTAM}]^{3+}$ complex.

On the other hand, titration of cytochrome $c_{3}$ with $[\mathrm{Gd}-\mathrm{DOTP}]^{5-}$ shows that there is a dramatic increase in the line width of the heme IV methyl M2 resonance of cytochrome $c_{3}$ and to a lesser extent of heme IV methyl M18 resonance, even at low ratios of $[\mathrm{Gd}-\mathrm{DOTP}]^{5-}$ to protein (Figure 3). A similar effect on the line width of these two methyls had been observed in the interaction between cytochrome $c_{3}$ and $\mathrm{Fe}$-rubredoxin. ${ }^{28}$ All other heme methyls resonances remain essentially unchanged in terms of line broadening, though some start to exhibit small shifts (with heme III's M2 being the most striking example).

The patch of positively charged lysines in the vicinity of heme IV enhances the probability of $[\mathrm{Gd}-\mathrm{DOTP}]^{5-}$ binding. The

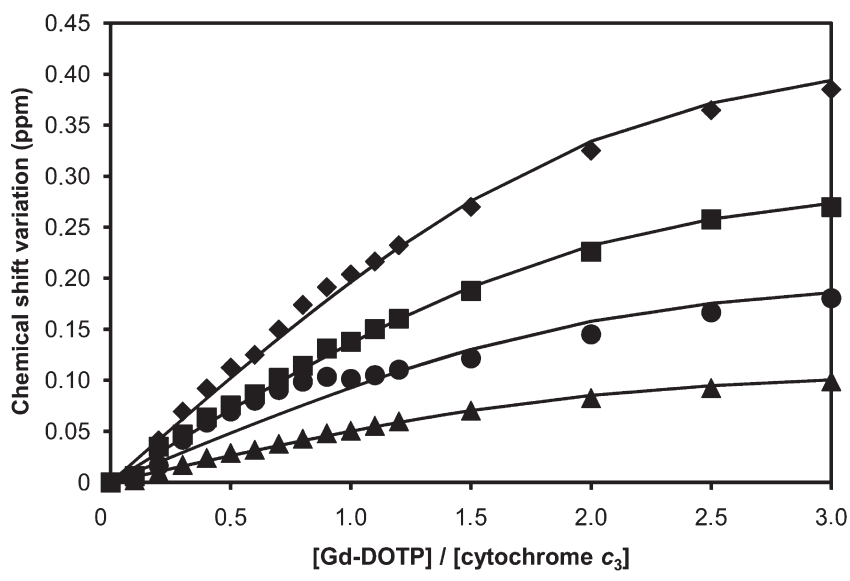

Figure 4. Chemical shift analysis of selected D. gigas cytochrome $c_{3}$ heme methyl resonances upon addition of $[\mathrm{Gd}-\mathrm{DOTP}]^{5-}$. Legend:

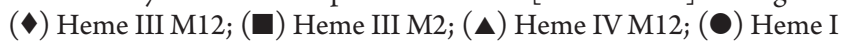
M7. An average value of $23 \pm 1 \mu \mathrm{M}$ was calculated for the $K_{\mathrm{d}}$ fitting the equation described in the Experimental Section considering a 2:1 binding equilibrium.

most solvent-exposed methyl groups of heme IV (M2 and M18) appear to suffer a more pronounced effect than the other assigned methyl resonance of heme IV (M12), which is buried in the protein core at a distance of $8 \AA$ from the surface. This effect becomes more pronounced as the titration progresses. Indeed, the $\mathrm{M} 2$ resonance becomes broadened beyond detection, while M18 experiences both line width broadening (of $110 \mathrm{~Hz}$ ) and a small chemical shift (data not shown, Supporting Information Table S1).

Several other methyl resonances belonging to other heme groups, but with special emphasis on the ones of heme III (resonances M2 and M12), also experience chemical shifts but without significant broadening (Figure 4 and Table S1, Supporting Information). This might be due, as postulated by us and other authors, ${ }^{28,47}$ to small conformational rearrangements of the cytochrome upon ligand binding, thus altering the chemical environment of the other heme groups. The lack of line broadening excludes direct binding of the paramagnetic probe in the vicinity of the other heme groups, as the presence of Gd implies that these chemical shifts cannot be due to long-range pseudocontact shifts. $^{48,49}$

The chemical shift variation enabled estimation of a value for the dissociation constant, $K_{\mathrm{d}}$, of $23 \pm 1 \mu \mathrm{M}$ for a $2: 1$ ([Gd-DOTP $]^{5-}$ :cytochrome $\left.c_{3}\right)$ binding model, considering two identical sites (Figure 4). This value is within the range of the dissociation constants determined for electrostatic-driven transient complexes. ${ }^{50,51}$

${ }^{15} \mathrm{~N}-\mathrm{Zn}$-Rubredoxin: An Acidic Target Protein. The effects of the two $\mathrm{Gd}$ probes on a small $\mathrm{Fe}$-protein, rubredoxin, were also examined. Figure 5 exhibits a partial area of the $\left[{ }^{1} \mathrm{H}\right.$, ${ }^{15} \mathrm{~N}$ ] HSQC spectrum of ${ }^{15} \mathrm{~N}-\mathrm{Zn}$-rubredoxin from $D$. gigas containing a significant number of the $\mathrm{HN}$ resonances of residues that belong to or are in the vicinity of the rubredoxin active center.

Addition of $[\mathrm{Gd}-\mathrm{DOTP}]^{5-}$ up to 0.5 equiv induced no significant changes in either chemical shifts or line widths of rubredoxin's resonances (Figure 5A, blue). Attending to the fact that this protein, at the working $\mathrm{pH}$, is mainly negatively charged with a hydrophobic patch near the metal center (Figure 2A), the results indicate that electrostatic repulsions with the negatively 


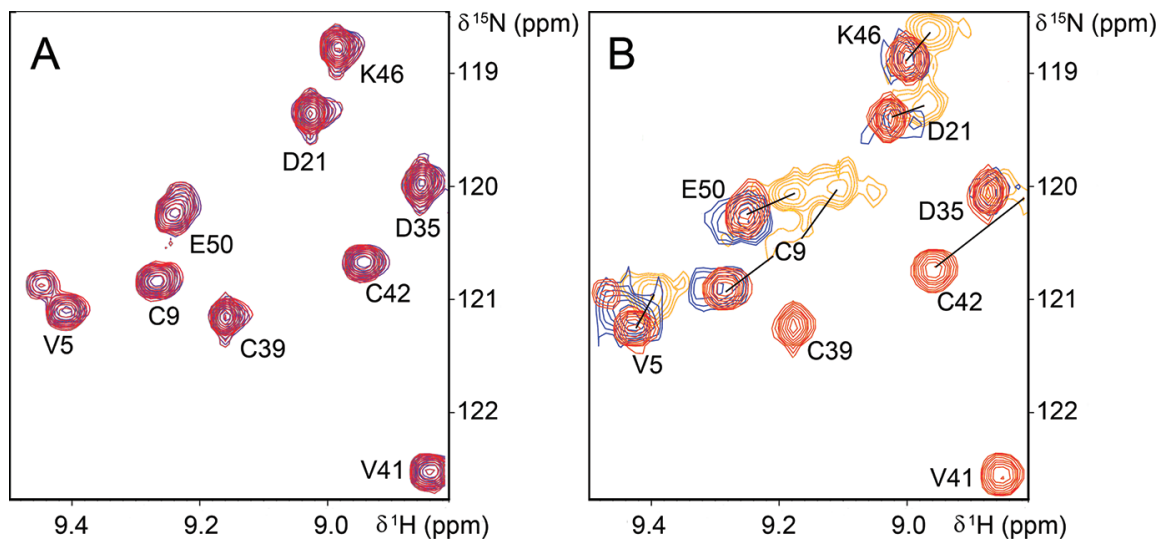

Figure 5. (A) $\left[{ }^{1} \mathrm{H},{ }^{15} \mathrm{~N}\right]$ HSQC spectra of the titration of $0.1 \mathrm{mM}$ D. gigas ${ }^{15} \mathrm{~N}-\mathrm{Zn}-$ rubredoxin with $[\mathrm{Gd}-\mathrm{DOTP}]^{5-}$ : (red) $[\mathrm{Gd}-\mathrm{DOTP}]^{5-} /[$ rubredoxin $]=0$; (blue) $[\mathrm{Gd}-\mathrm{DOTP}]^{5-} /[$ rubredoxin $]=0.50$. (B) $\left[{ }^{1} \mathrm{H},{ }^{15} \mathrm{~N}\right]$ HSQC spectra of the titration of $0.1 \mathrm{mM}$ D. gigas ${ }^{15} \mathrm{~N}-\mathrm{Zn}-$ rubredoxin with $[\mathrm{Gd}-\mathrm{DOTAM}]^{3+}:$ (red) $[\mathrm{Gd}-\mathrm{DOTAM}]^{3+} /[$ rubredoxin $]=0$; (blue) $[\mathrm{Gd}-\mathrm{DOTAM}]^{3+} /[$ rubredoxin $]=1.0 ;($ orange $)$ $[\mathrm{Gd}-\mathrm{DOTAM}]^{3+}:$ rubredoxin:cytochrome $c_{3}$ at $2: 1: 2$.

A
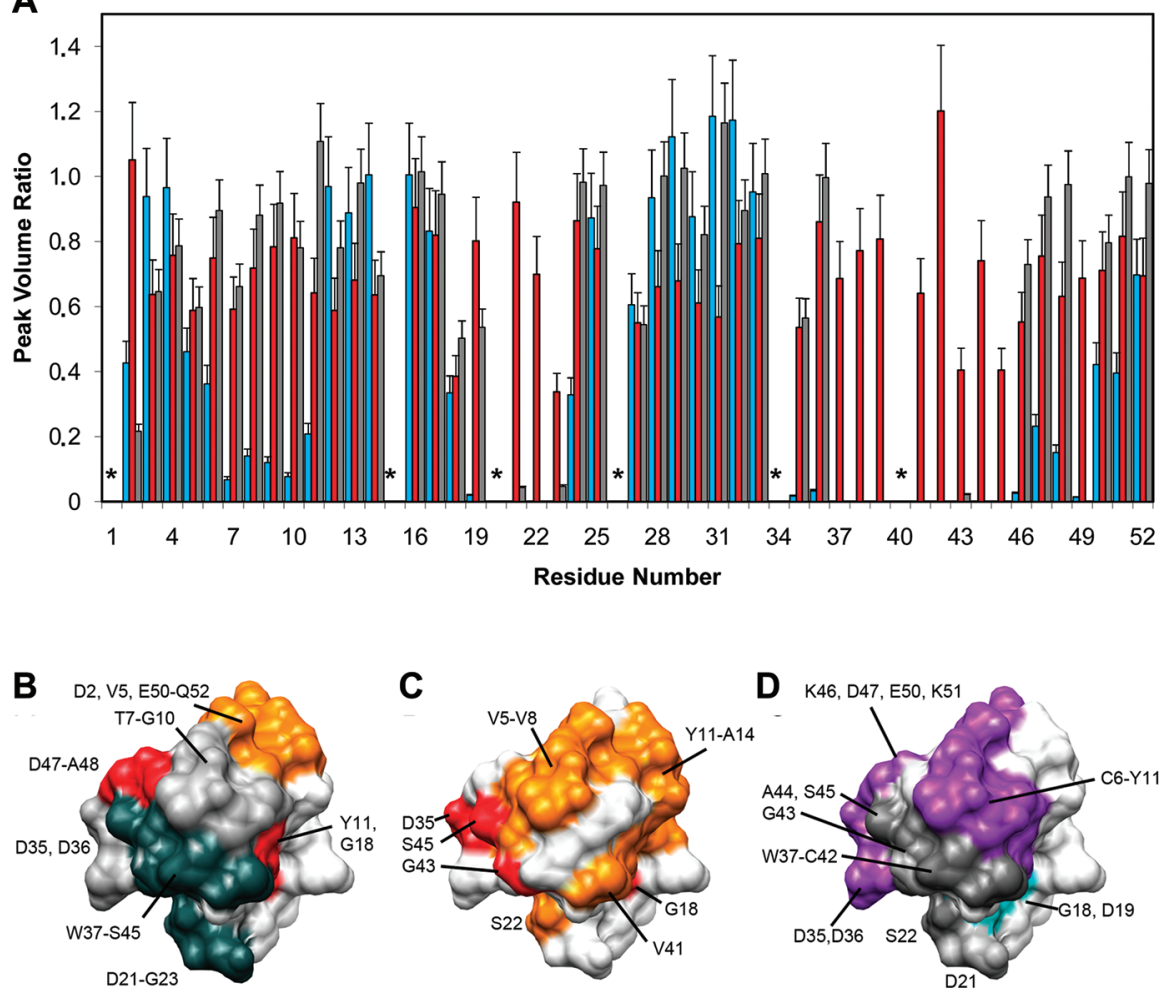

Figure 6. (A) Ratio of the peak volumes of rubredoxin amide resonances, comparing the free rubredoxin with rubredoxin in the presence of 2 mol equiv of $[\mathrm{Gd}-\mathrm{DOTAM}]^{3+}$ (blue), 2 mol equiv of cytochrome $c_{3}$ (red), and 2 mol equiv of $[\mathrm{Gd}-\mathrm{DOTAM}]^{3+}$ and 2 mol equiv of cytochrome $c_{3}$ (gray). Mapping of the rubredoxin surface according to the volume ratio presented in A. (B) Effect of $[\mathrm{Gd}-\mathrm{DOTAM}]^{3+}$ : Residues whose amide resonances have a decrease in volume ratio between $25 \%$ and $60 \%$ (orange), $60 \%$ and $90 \%$ (red), $90 \%$ and $100 \%$ (light gray), and 100\% (dark gray). (C) Effect of cytochrome $c_{3}$ : Residues whose amide resonances have a decrease in volume ratio between $20 \%$ and $40 \%$ (orange) and $40 \%$ and $60 \%$ (red). (D) Effect of cytochrome $c_{3}$ and $[\mathrm{Gd}-\mathrm{DOTAM}]^{3+}$ : Residues whose amide resonances have a significant recovery in their volume ratio (violet), small recovery (light blue), $4 \%$ recovery (light gray), and no recovery (dark gray).

charged DOTP-coordinated Gd(III) ion avoided any significant interaction (Figure 5A, blue).

Contrary to what was observed with $[\mathrm{Gd}-\mathrm{DOTP}]^{5-}$, the interaction with $[\mathrm{Gd}-\mathrm{DOTAM}]^{3+}$ resulted in significant spectral changes (Figure 5B, blue). It also becomes clear, just from spectral analysis, that resonances near the rubredoxin metal center become broadened, some beyond detection (Figure 5B, blue), and that, as expected, there are no significant chemical shift variation for any of the observed resonances due to the presence of this probe. ${ }^{6}$

Two different analyses of the effect of $[\mathrm{Gd}-\mathrm{DOTAM}]^{3+}$ addition were performed. In one case, the PRE rates were 

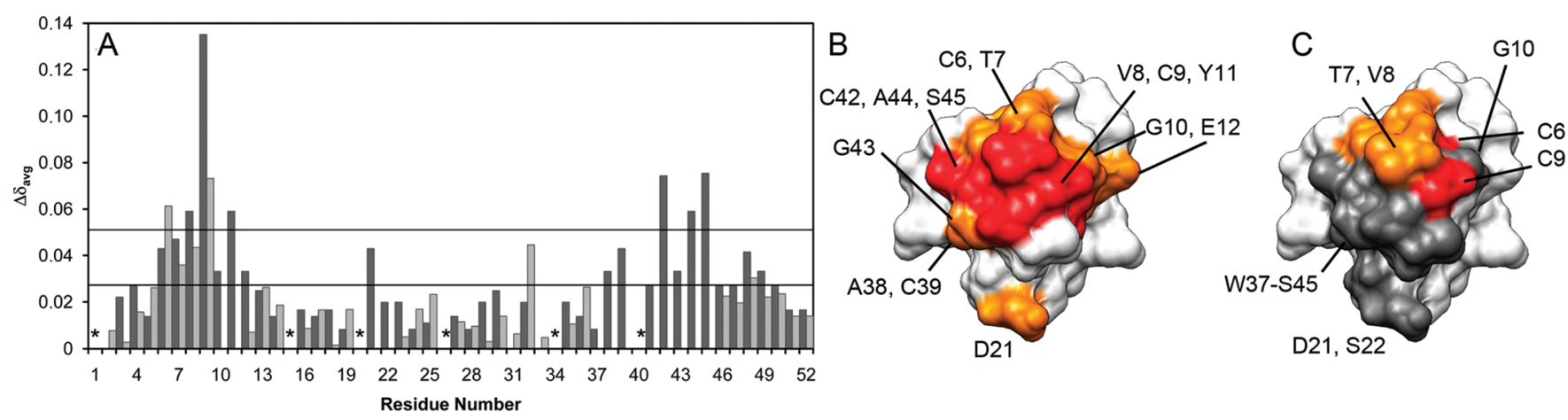

Figure 7. (A) Garrett plot of the titration of rubredoxin with 2 mol equiv of cytochrome $c_{3}$ (dark gray columns) alone or in the presence of 2 equiv of $[\mathrm{Gd}-\mathrm{DOTAM}]^{3+}$ (light gray columns). Asterisks indicate proline residues. Mapping of the most affected rubredoxin residues upon cytochrome $c_{3}$ binding alone (B) or in the presence of 2 equiv of $[\mathrm{Gd}-\mathrm{DOTAM}]^{3+}$ (C). Legend: (red) $\Delta \delta>0.05 \mathrm{ppm}$; (orange) $0.05 \mathrm{ppm}>\Delta \delta>0.025 \mathrm{ppm}$; (white) $\Delta \delta<0.025 \mathrm{ppm}$; (gray) residues broadened beyond detection.

determined (data not shown), indicating that for the $\mathrm{NH}$ resonances that were still observed this rate increased on average $2 \mathrm{~s}^{-1}$. This value is relatively small, compared with the ones reported in others studies using different Gd probes. ${ }^{52}$ Otting and co-workers determined PRE rates of $40 \mathrm{~s}^{-1}$ for residues away from the probe binding site and attributed this enhancement mainly to a nonspecific bulk solvent effect. The value determined in the present case is indeed smaller, which can be explained considering that this $\mathrm{Gd}$ probe, $[\mathrm{Gd}-\mathrm{DOTAM}]^{3+}$, has a remarkably slower water exchange rate ${ }^{53}$ than the one used in that other study, $\left[\mathrm{Gd}(\mathrm{DPA})_{3}\right]^{3-6}$. This in turn leads to a smaller fraction of water molecules being affected by the slow relaxation imposed by coordination to the Gd chelate, thus minimizing the bulk solvent effect that is brought upon by the exchange between the protein amide protons and the bulk water protons.

The other analysis of the paramagnetic effect was based on the volume ratio of the resonances and is presented in Figure 6. This analysis shows that the amide resonances of 11 residues (D21, S22, G23, W37, A38, C39, V41, C42, G43, A44, and S45, Figure 6) are broadened beyond detection. Moreover, although only one of these residues is negatively charged (D21), they are located near a negatively charged surface patch of rubredoxin (Figures 6B and 2A), and indeed, the intensity of the amide resonances of the other negatively charged residues decreases significantly in the presence of $[\mathrm{Gd}-\mathrm{DOTAM}]^{3+}$ (Figure $6 \mathrm{~A}$ and $6 \mathrm{~B}$ ). This indicates that the interaction between rubredoxin and this $\mathrm{Gd}$ probe is driven by electrostatic forces.

Nevertheless, contrary to what was observed in the interaction with cytochrome $c_{3}$, in the case of rubredoxin it is not possible to determine neither a binding affinity nor the complex stoichiometry. However, mapping of the residues, whose amide resonances experience the larger decrease in intensity, indicates that this interaction is specific and near a negatively charged surface patch (Figure 6A and 6B).

In order to determine whether $[\mathrm{Gd}-\mathrm{DOTAM}]^{3+}$ could be used as a relaxation probe to map and characterize the interface of protein complexes, the complex between rubredoxin and cytochrome $c_{3}$ was used as a case study. In Figure 5B the effects on rubredoxin amide resonances of adding 2 equiv of cytochrome $c_{3}$ (resonances in orange) to a solution of rubredoxin in the presence of 2 equiv of $[\mathrm{Gd}-\mathrm{DOTAM}]^{3+}$ are shown. These effects are either chemical shifts and/or recovery of signal intensity of some of the amide resonances (Figures 6 and 7). However, it is important to mention that the intensity of many of the resonances that were broadened beyond detection does not even partially recover, with the exception of the amide resonances of three residues, D21, G23, and G43, which recover about $4 \%$ of their original intensity.

The magnitude of the observed chemical shift difference on the rubredoxin amide resonances in the presence of 2 equiv of $[\mathrm{Gd}-\mathrm{DOTAM}]^{3+}$ and 2 equiv of cytochrome $c_{3}$ is similar to the one observed in the absence of lanthanide probe (Figure 7). ${ }^{28}$ As mentioned, the presence of 2 equiv of [Gd-DOTAM $]^{3+}$ does not induce by itself chemical shift perturbations on the rubredoxin amide resonances larger than $0.02 \mathrm{ppm}$ (data not shown), as expected for the interaction of such a lanthanide probe with an isotropic distribution of its unpaired electrons.

Therefore, attending to the fact that a chemical shift variation is observed on some resonances and others do not recover their intensity, it is proposed that cytochrome $c_{3}$ and $[\mathrm{Gd}-\mathrm{DOTAM}]^{3+}$ bind simultaneously to the rubredoxin surface. Indeed, both of these molecules might bind to rubredoxin with the same affinity (assuming a similar affinity as the one determined before for $[\mathrm{Gd}-\mathrm{DOTP}]^{5-}, 23 \pm 1$, and $25 \pm 2 \mu \mathrm{M}$ for cytochrome $\left.c_{3}{ }^{28}\right)$, and thus, even if competition for the same site is considered, the molar fraction of the bound molecule to rubredoxin would be 0.5 for both molecules, under these experimental conditions.

The ratio of the amide resonance integrals was analyzed and compared for three cases: (i) rubredoxin: $2[\mathrm{Gd}-\mathrm{DOTAM}]^{3+}$, (ii) rubredoxin:2cytochrome $c_{3}$, and (iii) rubredoxin: $2[\mathrm{Gd}-\mathrm{DOTAM}]^{3+}$ : 2 cytochrome $c_{3}$ (Figure 6). Analysis of the integral ratios and its mapping onto the rubredoxin surface indicates the following:

(i) Gd probe binds in a region close to the rubredoxin redox center with many resonances being broadened beyond detection (Figure 6B);

(ii) upon binding of cytochrome $c_{3}$ alone, the volume ratio of some rubredoxin amide resonances decreases $40-60 \%$ but most of them decrease only $20-40 \%$ and are not located in a specific surface region of rubredoxin (Figure 6C);

(iii) in the presence of both cytochrome $c_{3}$ and $[\mathrm{Gd}-$ DOTAM $]^{3+}$, some of the amide resonances recover, at least partially, the intensity that was lost due to the line width broadening imposed by $[\mathrm{Gd}-\mathrm{DOTAM}]^{3+}$ : C6-Y11, D35-D36, K46-D47, and E50-K51) (Figure 6D);

(iv) in the presence of both cytochrome $c_{3}$ and $[\mathrm{Gd}-$ DOTAM $]^{3+}$ some of the amide resonances that were 
not detected have a slight increase in their volume ratio (D21, G23, and G43) (Figure 6D).

Therefore, this analysis shows that although both molecules are binding simultaneously to rubredoxin, their binding sites are not superimposed (Figures 6 and 7). Moreover, the observation that some amide resonances are shielded upon binding of cytochrome $c_{3}$, in the presence of $[\mathrm{Gd}-\mathrm{DOTAM}]^{3+}$, enables a better definition of the rubredoxin surface to which it binds.

In conclusion, it can be proposed that cytochrome $c_{3}$ binds to rubredoxin near its redox center, in a region that is mainly hydrophobic, while $[\mathrm{Gd}-\mathrm{DOTAM}]^{3+}$ is positively charged and thus prefers binding to a more negatively surface patch.

In the present work the $\mathrm{Gd}$ probe binds to a specific region of the rubredoxin surface and thus is used in a different perspective and not to determine solvent-accessible regions or flexible regions as in other reports (reviewed in ref 54). The region that is more strongly affected is negatively charged, and binding of this Gd probe in the presence of cytochrome $c_{3}$ enabled a better definition of its binding site.

\section{CONCLUSIONS}

As hypothesized, the interaction between the negatively charged lanthanide chelator, $[\mathrm{Gd}-\mathrm{DOTP}]^{5-}$, and $\mathrm{Zn}-$ rubredoxin is negligible. This was expected since rubredoxin is extremely acidic, and thus, both the protein and $[\mathrm{Gd}-$ DOTP $]^{5-}$ have a negative net charge at the experimental $\mathrm{pH}$ values (7.6). Therefore, electrostatic repulsions dominate and prevent formation of any observable interaction. However, the interaction with cytochrome $c_{3}$ results in severe line broadening of resonances belonging to heme IV in a titration experiment, with the most exposed heme methyl, M2, being barely distinguishable from the baseline at a ratio of 0.08 and with M18 heme IV's methyl being less broadened $(110 \mathrm{~Hz})$. Chemical shifts occur in methyl resonances from the four heme groups but at larger extent than the ones from heme III and with a similar magnitude than the ones observed in the titration of cytochrome $c_{3}$ with rubredoxin. ${ }^{28}$ This might be due to conformational rearrangements, since $\mathrm{Gd}^{3+}$ does not induce pseudocontact shifts. Nevertheless, this effect enabled indirect determination of the dissociation constant of this probe. The estimated $K_{\mathrm{d}}$ of $23 \pm 1 \mu \mathrm{M}$ for a 2:1 complex stoichiometry, with two molecules of $\mathrm{Gd}^{3+}$ probe binding to cytochrome $c_{3}$, is in line with this complex being an electrostatic-driven transient complex. Thus, it is proposed that the $[\mathrm{Gd}-\mathrm{DOTP}]^{5-}$ probe can be used as a cosolute to identify positively charged patches at protein surfaces.

For the positively charged relaxation probe, $[\mathrm{Gd}-\mathrm{DOTAM}]^{3+}$, the interaction with cytochrome $c_{3}$ is remarkably weak when compared to the chemical shift changes brought upon by $[\mathrm{Gd}-\mathrm{DOTP}]^{5-}$. Moreover, no significant line width broadening occurs, which also reinforces the observation that there is no binding of $[\mathrm{Gd}-\mathrm{DOTAM}]^{3+}$ to cytochrome $c_{3}$ near any of the heme groups. However, the interaction of $[\mathrm{Gd}-\mathrm{DOTAM}]^{3+}$ with $\mathrm{Zn}$-rubredoxin induced broadening on selected resonances near the active center, in some cases to the point of no detection. Addition of cytochrome $c_{3}$ partly reversed this effect, with some of the amide resonances recovering their intensity, but it was also observed that other resonances experience chemical shift perturbation in a similar magnitude to the ones observed in the presence of cytochrome $c_{3}$ alone. Therefore, it is proposed that both molecules bind simultaneously to rubredoxin but at a different site. Analysis of the shielded resonances in the presence of cytochrome $c_{3}$ enabled a better definition of its binding site.

In conclusion, the results obtained illustrate the use of $[\mathrm{Gd}-\mathrm{DOTAM}]^{3+}$ as a relaxation probe in the characterization of protein complexes.

\section{ASSOCIATED CONTENT}

S Supporting Information. Listings of the induced chemical shift perturbation on cytochrome $c_{3}$ heme methyl resonances caused by $\mathrm{Fe}-$ rubredoxin and $[\mathrm{Gd}-\mathrm{DOTP}]^{5-}$. This material is available free of charge via the Internet at http://pubs.acs.org.

\section{AUTHOR INFORMATION}

\section{Corresponding Author}

*Phone: + 351212948 382. Fax: + 351212948 550. E-mail: jose. moura@dq.fct.unl.pt (J.J.G.M.); srp@dq.fct.unl.pt (S.R.P.).

\section{ACKNOWLEDGMENT}

The authors thank Fundação para a Ciência e Tecnologia for the Ph.D. scholarship granted to R.M.A. (SFRH/BD/25342/ 2005) and C.F.G.C.G. for project PTDC/QUI/70063/2006. We acknowledge LabRMN at FCT-UNL and Rede Nacional de RMN for access to the facilities. The NMR spectrometers are part of the National NMR Network and were purchased in the framework of the National Programme for Scientific Reequipment, contract REDE/1517/RMN/2005, with funds from POCI 2010 (FEDER) and Fundação para a Ciência e a Tecnologia (FCT).

\section{REFERENCES}

(1) Bertini, I.; Luchinat, C. Coord. Chem. Rev. 1996, 150, 77-110.

(2) Bertini, I.; Luchinat, C.; Parigi, G. Solution NMR of Paramagnetic Molecules. In Current Methods in Inorganic Chemstry; Elsevier: Amsterdam, 2001; Vol. 2.

(3) Peters, J. A.; Huskens, J.; Raber, D. J. Prog. Nucl. Magn. Reson. Spectrosc. 1996, 28, 283-350.

(4) Allegrozzi, M.; Bertini, I.; Janik, M. B.; Lee, M.-Y.; Luchinat, C. J. Am. Chem. Soc. 2000, 122 (17), 4154-4161.

(5) Clore, G. M.; Iwahara, J. Chem. Rev. 2009, 109 (9), 4108-4139.

(6) Yagi, H.; Loscha, K. V.; Su, X. C.; Stanton-Cook, M.; Huber, T.; Otting, G. J. Biomol. NMR 2010, 47 (2), 143-153.

(7) Bertini, I.; Janik, M. B.; Lee, Y. M.; Luchinat, C.; Rosato, A. J. Am. Chem. Soc. 2001, 123 (18), 4181-4188.

(8) Geraldes, C. F.; Luchinat, C. Met. Ions Biol. Syst. 2003, 40, 513-588.

(9) Banci, L.; Bertini, I.; Bren, M. A.; Cremonini, M. A.; Gray, H. B.; Luchinat, C.; Turano, P. J. Biol. Inorg. Chem. 1996, 1, 117-126.

(10) Bentrop, D.; Bertini, I.; Luchinat, C.; Nitschke, W.; Muhlenhoff, U. Biochemistry 1997, 36 (44), 13629-13637.

(11) Bertini, I.; Felli, I. C.; Luchinat, C. J. Biomol. NMR 2000, 18, 347-355.

(12) Bertini, I.; Janik, M. B.; Liu, G.; Luchinat, C.; Rosato, A. J. Magn. Reson. 2001, 148 (1), 23-30.

(13) Pintacuda, G.; Otting, G. J. Am. Chem. Soc. 2002, 124 (3), 372-373.

(14) Pintacuda, G.; Park, A. Y.; Keniry, M. A.; Dixon, N. E.; Otting, G. J. Am. Chem. Soc. 2006, 128 (11), 3696-3702.

(15) Su, X. C.; Liang, H.; Loscha, K. V.; Otting, G. J. Am. Chem. Soc. 2009, 131 (30), 10352-10353.

(16) Clore, G. M.; Tang, C.; Iwahara, J. Curr. Opin. Struct. Biol. 2007, $17(5), 603-616$. 
(17) Kleckner, I. R.; Foster, M. P. Biochim. Biophys. Acta 2011, 1814 (8), 942-968.

(18) Saio, T.; Yokochi, M.; Kumeta, H.; Inagaki, F. J. Biomol. NMR 2010, 46 (4), 271-280.

(19) Donaldson, L. W.; Skrynnikov, N. R.; Choy, W. Y.; Muhandiram, D. R.; Sarkar, B.; Forman-Kay, J. D.; Kay, L. E. J. Am. Chem. Soc. 2001, 123 (40), 9843-9847.

(20) Feeney, J.; Birdsall, B.; Bradbury, A. F.; Biekofsky, R. R.; Bayley, P. M. J. Biomol. NMR 2001, 21 (1), 41-48.

(21) Ikegami, T.; Verdier, L.; Sakhaii, P.; Grimme, S.; Pescatore, B.; Saxena, K.; Fiebig, K. M.; Griesinger, C. J. Biomol. NMR 2004, 29 (3), 339-349.

(22) Martin, L. J.; Hahnke, M. J.; Nitz, M.; Wohnert, J.; Silvaggi, N. R.; Allen, K. N.; Schwalbe, H.; Imperiali, B. J. Am. Chem. Soc. 2007, 129 (22), 7106-7113.

(23) Pintacuda, G.; John, M.; Su, X. C.; Otting, G. Acc. Chem. Res. 2007, 40 (3), 206-212.

(24) Prudencio, M.; Rohovec, J.; Peters, J. A.; Tocheva, E.; Boulanger, M. J.; Murphy, M. E.; Hupkes, H. J.; Kosters, W.; Impagliazzo, A.; Ubbink, M. Chemistry 2004, 10 (13), 3252-3260.

(25) Su, X. C.; Otting, G. J. Biomol. NMR 2010, 46 (1), 101-112.

(26) Vlasie, M. D.; Comuzzi, C.; van den Nieuwendijk, A. M.; Prudencio, M.; Overhand, M.; Ubbink, M. Chemistry 2007, 13 (6), 1715-1723.

(27) Xu, X.; Keizers, P. H.; Reinle, W.; Hannemann, F.; Bernhardt, R.; Ubbink, M. J. Biomol. NMR 2009, 43 (4), 247-254.

(28) Almeida, R. M.; Pauleta, S. R.; Moura, I.; Moura, J. J. G. J. Inorg. Biochem. 2009, 103 (9), 1245-1253.

(29) Tam, S. C.; Williams, R. J. P. Struct. Bonding (Berlin) 1985, 63, 103-151.

(30) Arean, C. O.; Moore, G. R.; Williams, G.; Williams, R. J. P. Eur. J. Biochem. 1988, 173 (3), 607-615.

(31) Eley, C. G.; Moore, G. R.; Williams, G.; Williams, R. J. P. Eur. J. Biochem. 1982, 124 (2), 295-303.

(32) Dalgarno, D. C.; Price, H. P.; Levine, B. A.; Trayer, I. P. Biochim. Biophys. Acta 1982, 707, 81-88.

(33) Liepinsh, E.; Baryshev, M.; Sharipo, A.; Ingelman-Sundberg, M.; Otting, G.; Mkrtchian, S. Structure 2001, 9 (6), 457-4571.

(34) Petros, A. M.; Mueller, L.; Kopple, K. D. Biochemistry 1990, 29 (43), 10041-10048.

(35) Sattler, M.; Fesik, S. J. Am. Chem. Soc. 1997, 119 (33), $7885-7886$.

(36) Aime, S.; D’Amelio, N.; Fragai, M.; Lee, Y. M.; Luchinat, C.; Terreno, E.; Valensin, G. J. Biol. Inorg. Chem. 2002, 7 (6), 617-622.

(37) Dick, L. R.; Geraldes, C. F.; Sherry, A. D.; Gray, C. W.; Gray, D. M. Biochemistry 1989, 28 (19), 7896-7904.

(38) Folkers, P. J.; van Duynhoven, J. P.; van Lieshout, H. T.; Harmsen, B. J.; van Boom, J. H.; Tesser, G. I.; Konings, R. N.; Hilbers, C. W. Biochemistry 1993, 32 (36), 9407-9416.

(39) Aime, S.; Barge, A.; Botta, M.; de Sousa, A. S.; Parker, D. Angew. Chem., Int. Ed 1998, 37, 2673-2675.

(40) Desreux, J. F. Inorg. Chem. 1980, 19 (5), 1319-1324.

(41) Geraldes, C. F. C. G.; Sherry, A. D.; Kiefer, G. E. J. Magn. Reson. 1992, 97, 290-304.

(42) Lazar, I.; Hrncir, D. C.; Kim, W. D.; Kiefer, G. E.; Sherry, A. D. Inorg. Chem. 1992, 31 (21), 4422-4424.

(43) Barge, A.; Cravotto, G.; Gianolio, E.; Fedeli, F. Contrast Media Mol. Imaging 2006, 1 (5), 184-188.

(44) Garrett, D. S.; Seok, Y. J.; Peterkofsky, A.; Clore, G. M.; Gronenborn, A. M. Biochemistry 1997, 36 (15), 4393-4398.

(45) Berger, S.; Braun, S., 200 and More NMR Experiments: A Practical Course; Wiley-VCH: New York, 2004; p 854.

(46) Trott, O.; Olson, A. J. J. Comput. Chem. 2010, 31 (2), 455-461.

(47) Stewart, D. E.; Wampler, J. E. Proteins 1991, 11 (2), 142-152.

(48) Bertini, I.; Turano, P.; Vila, A. J. Chem. Rev. 1993, 93 (8), $2833-2932$.

(49) Otting, G. J. Biomol. NMR 2008, 42 (1), 1-9.

(50) Crowley, P. B.; Carrondo, M. A. Proteins 2004, 55 (3), 603-612.
(51) Lo Conte, L.; Chothia, C.; Janin, J. J. Mol. Biol. 1999, 285 (5), 2177-2198.

(52) Caravan, P. Chem. Soc. Rev. 2006, 35 (6), 512-523.

(53) Thompson, A. L.; Parker, D.; Fulton, D. A.; Howard, J. A.; Pandya, S. U.; Puschmann, H.; Senanayake, K.; Stenson, P. A.; Badari, A.; Botta, M.; Avedano, S.; Aime, S. Dalton Trans. 2006, 47, 5605-5616.

(54) Otting, G. Annu. Rev. Biophys. 2010, 39, 387-405.

(55) Pettersen, E. F.; Goddard, T. D.; Huang, C. C.; Couch, G. S.; Greenblatt, D. M.; Meng, E. C.; Ferrin, T. E. J. Comput. Chem. 2004, 25 (13), 1605-1612.

(56) Cornell, W. D.; Cieplak, P.; Bayly, C. I.; Gould, I. R.; Merz, K. M., Jr; Ferguson, D. M.; Spellmeyer, D. C.; Fox, T.; Caldwell, J. W.; Kollman, P. A. J. Am. Chem. Soc. 1995, 117 (19), 5179-5197.

(57) Wang, J.; Wang, W.; Kollman, P. A.; Case, D. A. J. Mol. Graph. Model 2006, 25 (2), 247-260. 